Voix et Images

volxetimages

\title{
La lutte avec l'ange. Le corps à corps avec le Nom dans la prose de Yolande Villemaire. De la Vie en prose à la Constellation du Cygne.
}

\section{Anne Élaine Cliche}

Volume 11, numéro 3 (33), printemps 1986

Yolande Villemaire

URI : https://id.erudit.org/iderudit/200579ar

DOI : https://doi.org/10.7202/200579ar

Aller au sommaire du numéro

\section{Éditeur(s)}

Université du Québec à Montréal

\section{ISSN}

0318-9201 (imprimé)

1705-933X (numérique)

Découvrir la revue

\section{Citer cet article}

Cliche, A. É. (1986). La lutte avec l'ange. Le corps à corps avec le Nom dans la prose de Yolande Villemaire. De la Vie en prose à la Constellation du Cygne..

Voix et Images, 11(3), 440-453. https://doi.org/10.7202/200579ar 


\title{
La lutte avec l'ange. Le corps à corps avec le Nom dans la prose de Yolande Villemaire. De la Vie en prose à la Constellation du Cygne.
}

\author{
par Anne Êlaine Cliche, Université d'Ottawa
}

La littérature est une imposture généralisée en ceci que, ne s'adressant à personne, elle ne compose pas. Foyer de tous les noms, elle est sans nom - toujours en train de nommer. Comme fonction d'interruption, elle infirme le lien social de la communauté et, par cette intermission, elle affirme la permanence de l'interpellation qui n'en finit plus d'entendre le bruit du monde par où quelqu'un passe, incognito, occupant toutes les places sans en retenir aucune, pour rentrer dans son nom ${ }^{1}$.

La prose est ce qui, du nom, y retourne pour en détourner le propre, pour en déporter l'émergence de l'un à l'autre. Écriture de la lettre (lettre d'amour) dont la signature en bas de page décline une identité en suspension où le corps social s'affiche en répétant le passage de celui ou celle qui s'efface en se signant. Le nom fait signe en "clignotant» une singularité dans laquelle il semble précisément qu'il n'y ait plus personne: signal du discontinu, mouvement d'un «je» qui n'en finit pas de devenir un «je». C'est l'évidement de la langue qui parle à la première personne.

Si le nom suppose quelqu'un, un être «parlable», sa profération (l'appel) énonce une identité en creux dont l'écriture impose le retournement parodique. C'est là que se trace la "rencontre» de l'écrit et du réel, de la "prose» et de la "vie»» ${ }^{2}$ : dans la désertation du nom. La signature de la lettre n'est alors que la "constellation du signe", le point de convergence où le sujet est toujours reconnu et toujours inconnaissable.

Le nom inaugure l'errance, le "passage incognito", la quête démultipliée d'un sujet dont la constitution est justement dans cet investissement impossible du nom. Ainsi, "la vie en prose" pourrait bien être la formulation de cette quête du nom, de l'écrit, et de sa mise en déroute. Rencontre (manquée) $\mathrm{du}$ réel et du verbe dans ce point de fuite où ni l'un ni l'autre ne réalise sa clôture et où le sujet ne peut émerger qu'à s'inscrire dans la double fracture du nom et du corps.

La vie en prose, parce que la distinction n'existe pas... Si elle $n^{\prime}$ existe pas, elle existe, pourtant, simultanément ${ }^{3}$. 
Aussi, au détour de l'écrit, c'est l'écriture qui opère l'épreuve du réel et de son "désert", la parole comme le nom ne venant jamais combler le manque à être (manque de vie) du sujet:

Je manque peut-être un peu de vie depuis que je me suis enfermée à double tour dans la prose 4 .

Cette problématique est donc l'acte même du premier "grand" roman de Villemaire. Aucune plénitude n'est possible dans cette prose où la mise en circulation de la «lettre» est d'abord une mise en scène de la circularité du nom dont le centre vide répète la perte de l'identité des sujets et la clôture du subjectif dans l'imaginaire de la fiction:

... tantôt, tout le roman m'est apparu comme l'histoire de la passion de Nane Yelle pour Rael, personnage énigmatique qui, dans la vie réelle s'appelle Réal et dont elle vient aujourd'hui de recevoir une lettre... je dis que (...) personne n'ose s'appeler par son vrai nom, que f̧a fait tellement longtemps qu'on nage en pleine fiction... ${ }^{5}$

Écrire serait peut-être justement reconnaître la blessure d'un corps de désir qui appelle pour mieux dé-nommer; passage (à vide) du corps en langue( $s$ ) où le nom s'oublie en se donnant comme toujours autre. L'écriture, dans la Vie en prose, s'affirme ainsi en opérant le dérapage du nom (de la signature) et de l'identité, par évocations multiples où le corps s'écri(e)t en morcelé. Elle rompt le fil de la fiction en perdant celui ou celle qui s'y abandonne, en l'obligeant à rejoindre le centre insaisissable des voix qui la fondent. Cette écriture met en scène l'infini des possibilités narratives et maintient en filigrane son procès. Ce procès qui sous-tend toute l'élaboration formelle du roman pourrait bien s'énoncer sous la forme d'un questionnement: Comment échapper à la prose en sachant qu'il est impossible d'y échapper? Réponse: En l'accélérant.

C'est donc la question de l'accélération, avec sa multiplication de réseaux de voix et de doubles, qui se trouve mise en jeu par l'écriture. Réseaux où s'échangent des voix, des énoncés, des personnages, des noms, orbite des mondes et des désirs qui formule l'accélération non plus en termes de vitesse mais en termes d'affolement, de décentrement où le temps devient réversible et perd toute référence linéaire pour inscrire la simultanéité du même et de l'autre. Inscription du réel dont le surgissement, toujours menacé, se trouve tout entier dans ce temps d'apparition qui est à la fois celui de sa disparition.

L'écriture de la lettre passe d'une main à l'autre, investissant à tour de rôle un "je» dont le destinataire n'est jamais que la lettre elle-même, c'est-àdire l'ouverture de l'autre dans le semblable ${ }^{6}$. Mais cette répétition, cet excès de redoublements démultipliant l'instance narratrice et l'acte de nomination, finit par mettre à l'épreuve (faire éprouver) l'irrépétable. Ce que le sujet demande, ce qu'il réclame, ce qu'il tente de faire de l'écrit, c'est la représentation du réel. L'affolement du double répète ce qui, dans la langue, se "règle» (la quête du sujet ou de la fiction), mais c'est pour rendre le réel (le manque- 
ment de la langue) à sa non-représentabilité?. L'irrépétable est dans cet écart où les mots manquent, où cela fait silence de ne pouvoir se dire.

De plus, chez Villemaire, l'excès comme surgissement du silence (incision du réel dans la fiction) s'affirme particulièrement dans la figure de l'ange. L'ange de Villemaire n'est donc plus la métaphore de la plénitude androgyne totalisant les deux sexes dans la fusion des complémentarités. La figure du grand mythe de l'Un, du Même, surgit pour s'excéder dans sa propre mise en jeu, révélant son envers insaisissable, une impossibilité qui rejoint la configuration du nom. L'ange participe du différé de la lettre, de l'errance métonymique: il en est l'instaurateur toujours fuyant et toujours appelé.

Le nom de l'ange résonne à travers toute la Vie en prose ${ }^{8}$, occupe toutes les voix (amants, frères, jumeaux, narratrices, jumelles) jusqu'à la dérision qui le relance en point de fuite du sujet acharné à "entrer» dans son nom. L'ange ne se fige jamais longtemps dans l'illusoire de la représentation. La fiction (fixion) de l'ange n'est pas à lire mais, au contraire, finit par s'assimiler à la prose qui, par accélération de l'invocation, creuse un point centrifuge où bascule sa possibilité même. La figure androgyne est ainsi rompue à son intersexualité non complémentaire (devenue figure du non rapport), et rend la lettre à son illisibilité, à sa fin de non recevoir. Ainsi, la lecture est toujours en souffrance de la lettre, celle-ci faisant constamment retour à l'envoyeur puisqu'il n'y a pas d'autre destinataire que ce singulier pluriel en train de se prendre en compte dans les renvois 9 .

Aussi ce Rael, dont le nom fait signe et à partir duquel la prose s'accélère de ne pouvoir le rejoindre, est-il le corps d'amour qui tisse des ponts entre la fiction du nom et son manque à nommer, entre prose et vie. Cet innommable du réel est peut-être ce qui travaille au plus profond l'écriture de Villemaire. En effet, si l'on aborde cet autre roman qu'est la Constellation du Cygne ${ }^{10}$. c'est à une écriture transformée, transfigurée, dépouillée, que nous avons affaire, mais celle-ci s'articule encore sur un fond de silence qui s'y joue. Et cette écriture nouvelle permet de saisir à quel point les figures de l'excès de la Vie en prose (anges, voix multiples, répétitions, retours) ne sont que les envers d'une absence dont se marque l'acte toujours renouvelé de la nomination.

$\mathrm{Si}$, dans le premier roman, le nom "vrille» la fiction en se sursignifiant, dans la Constellation on est à l'écoute de corps, de voix, de noms qui se donnent dans l'évidence (l'évidement) de leur blessure. Passage du signe au cygne: deux champs (chants) ou deux modes de mise à l'épreuve du réel, l'excès de l'un rejoignant inévitablement la mort de l'autre. Ce dernier roman dont la division en chapitres se fait sous la bannière du nom propre (Celia Rosenberg, Karl-Heinz Hausen, Gabrielle Lévy, Piotr Jalski, Auschwitz et La constellation du Cygne) s'articule autour d'un double ravissement, celui de la jouissance et celui d'une échappée constante du savoir. 
Ce temps du ravissement est au centre du personnage de Celia Rosenberg, à la fois comme son "transport" érotique et son inaccessibilité au «lieu» de la vérité. Celia ne sait pas, ne connaît pas, n'entend pas la langue de celui qui, soudain, vient provoquer sa jouissance dans un érotisme dont il semble que seul le nom soit garant:

Karl Heinz Hausen, pense Celia Rosenberg, Karl-Heinz Hausen. Elle ferme les yeux de joie tandis qu'une luminescence la nimbe de blancheur.

Mais vous êtes là, dit-elle en se tournant vers lui, Karl-Heinz Hausen. Elle répète: Karl-Heinz Hausen. J'aime votre nom, Karl-Heinz Hausen.

La Constellation donne libre cours à un corps qui s'écrit et se décrit (décrie) dans une érotique où vient se greffer un dense univers symbolique dont l'impuissance à révéler le «vrai» ne fait que remettre en scène l'interdit de la représentation. L'érotique se joue donc ici sur cette double scène de l'anéantissement: celui, singulier. du sujet dans la jouissance, et celui, collectif, des Juifs dans l'holocauste nazi (dont l'imminence s'actualise dans la liaison entre Celia et Karl-Heinz). Plus précisément, l'érotique surgit d'une écriture qui s'articule au lieu même de l'impossible, de l'indicible et de la mort pour en cerner davantage les formes creuses.

La figure centrale du cygne traverse le roman, affirmant la double instance sexuelle et mortuaire dans son indénouable intrication. La «constellation du Cygne" exprime donc, en même temps qu'un amas d'étoiles (lumière blanche), cette fusion du désir et de la mort où le cou blanc et long du cygne devient le signifiant premier. Cou à blesser, à trancher, à mordiller, à tordre dans le meurtre comme dans l'amour.

Sur les bords de la Vistule, un couple de cygnes, toutes plumes ébouriffées, se déchirent à coups de becs agressifs, dans un ballet muèt pathétique. L'un deux est blessé au cou et le sang commence à maculer son plumage blanc.

Brusquement Piotr Jalski (...) met ses grosses mains autour du cou de Celia Rosenherg et la serre à l'étouffer (...) il se met à mordiller ce cou qu'il vient de meurtrir.

Vous avez le cou blanc, tellement blanc. La peau en est si fine. - J'aime quand vous mordez mon cou, Piotr Jalski. 
Le cygne, parce qu'il est marqué de l'érotisme, devient le signifiant du manque réel dont la mort est le centre incontournable. C'est à ce double rapport (mort/Éros) que renvoient les nombreuses occurrences de la blancheur et de la lumière.

$\mathrm{La}$ "constellation du Cygne» est ainsi ce lieu hors-lieu d'où l'écrit se constitue. Cet espace indésignable rejoint alors l'inimaginable de l'holocauste, son déni par Celia, le défaut de savoir où elle se situe quant à l'extermination de sa «race». Les noms propres qui ponctuent le texte ne sont plus qu'effets de signifiance; ils apparaissent comme le négatif du génocide, négatif de cette lacune qu'est la vérité indicible. Juifs, allemands, polonais, français, les noms propres ne sont plus que fantômes, signature de la langue elle-même, signifiants purs. Ils marquent du sceau textuel le rapport sexuel impossible.

D'autre part, pour Celia, les noms appellent le mouvement d'une réminiscence opaque, occultée ou occulte. Dans le nom, plus rien n'a lieu alors que la mémoire. Celia Rosenberg est au point d'aveuglement, dans une impuissance physique à connaître la vérité de ce qui est en train de s'accomplir. D'une part parce qu'elle est la visée du plan d'extermination des Juifs qui commence par un leurre centré sur la dénomination, par un transfert de signes, et, d'autre part parce qu'elle participe entièrement de ce transfert de signes, de cette occultation. Elle est au point de disparition du sens et de l'histoire. Celia Rosenberg se maintient dans le non savoir, dans la tache aveugle de cette vision impraticable d'être devenue voyance:

Elle voudrait tout expliquer mais elle ne sait pas comment. Elle ne sait pas.

(ils) semblent tous vouloir lui parler en même temps de quelque chose dont elle ne veut surtout pas entendre parler.

Celia Rosenberg ne veut pas comprendre ce que signifie porter l'étoile jaune. Celia Rosenberg ne veut pas comprendre ce que signifie être juive et aimer à la folie un officier nazi. Celia Rosenberg ne veut pas comprendre.

- Gabrielle Lévy disait qu'il me faudrait trouver le canal 55.

- Le canal 55 ?

- Je ne sais pas ce que c'est. Quelque chose comme un rite de passage, je crois.

- C'est le nom de code que nous utilisions pour les passages en Suisse.

- Ah? Je ne savais pas. Mais il y a aussi une interprétation cabalistique, $j$ 'en suis certaine. 
Celia est occultée, mais cette occultation n'est que le support d'un occultisme dont les signes multiples naissent du regard des amants. Les figures ailées qui surgissent et se teintent de l'œil vert ou bleu hantent la conscience et les rêves de Celia Rosenberg. Comme si la vision du "vrai" (qui est propre à Karl Heinz Hausen, à Piotr Jalski, à Klauss Schule) laissait la trace d'un autre savoir, initiatique, dont Celia s'investit pour se soumettre à l'opacité du réel. L'inimaginable, parce qu'il n'a d'autre existence possible qu'imaginaire, retourne donc à la fiction du symbole. Les figures "astrales" sont alors autant de hiéroglyphes demeurés indéchiffrés, signes en creux dont l'impossible signification ne fait que signaler le versant innommé du réel ${ }^{11}$. Ainsi,

Celia Rosenberg voit très nettement le rictus de la magicienne à la peau verte dans les yeux fous de Karl-Heinz Hausen...

alors que les yeux bleus de Piotr suscitent l'apparition d'un ange bleu aux ailes moelleuses (p. 150). Ceci conduit à dire que si la réalité comme effet de langage appartient aussi à l'axe de la fiction et traverse aussi bien la vérité que le mensonge, les hiéroglyphes que constituent les figures "angéliques» tiennent, eux, du réel. C'est dire qu'ils sont la trace de l'impossible du langage. La magicienne à la peau verte, le cheval bleu, les hippocampes ailés (p. 36), les anges bleus, blancs et noirs (p. 150, 158, 175-177) viennent former des réseaux qui démultiplient l'enjeu central de la "constellation du Cygne»: celui d'un clignotement perpétuel de la jouissance et de la mort, du corps et du cosmique.

Celia Rosenberg se trouve au point d'intersection de ces deux axes de l'occultation et de l'occultisme, dans ce "point mort» où ils s'abolissent de n'être que des leurres. Axes croisés ${ }^{12}$ dont la mise en orbite (le texte) creuse l'inscription du réel. Écrire le réel, pourrait-on dire, c'est creuser, manquer à la réalité. C'est ce que la "constellation du Cygne" telle que le texte la met en jeu, permet de reconnaître: 


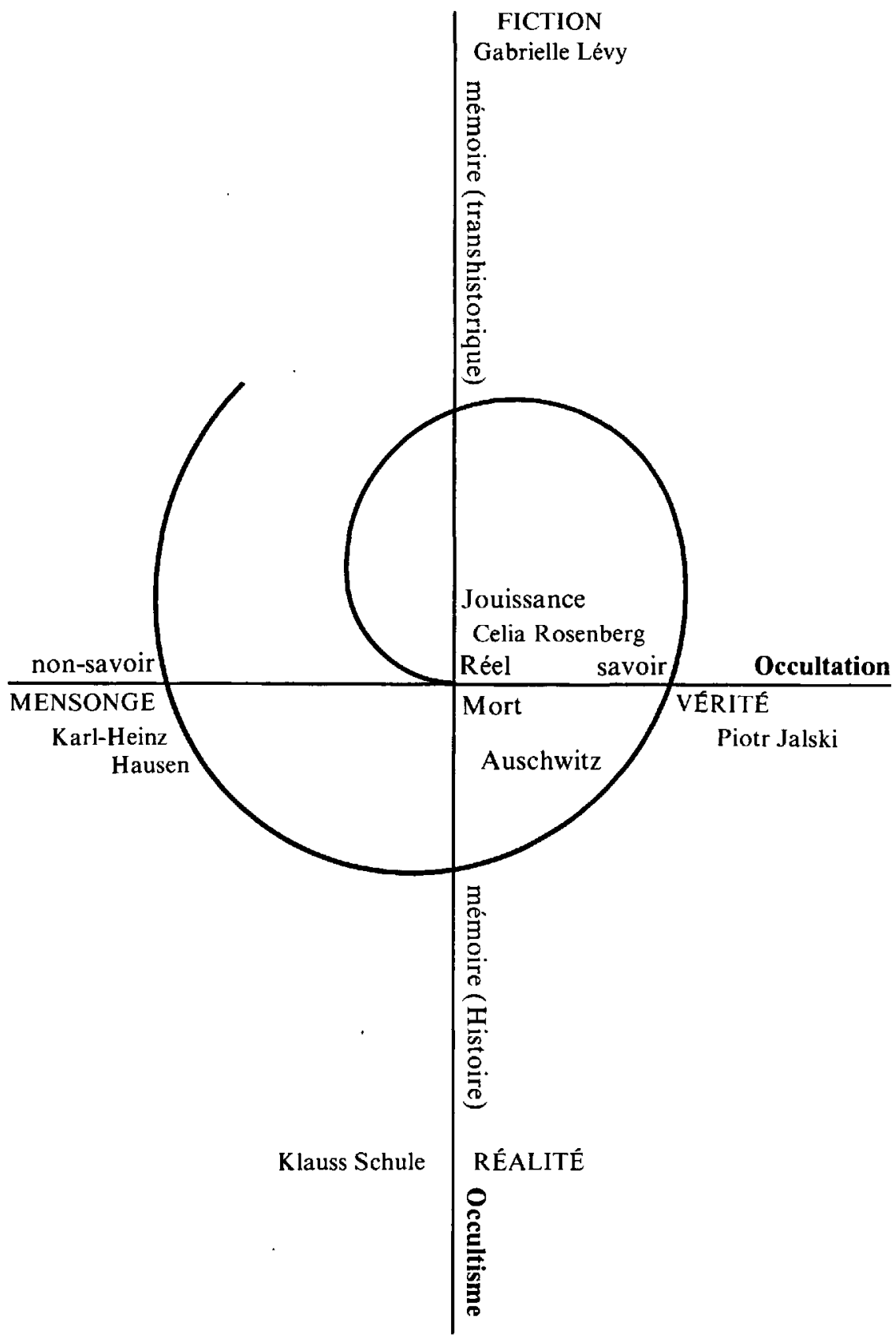

La Constellation du Cygne 
Au point de cette rencontre (manquée) des deux axes, se dérobe l'indicible (holocauste, jouissance) d'où rien d'autre ne peut surgir que la mémoire. Mémoire qui ramène la question du double dans sa problématique dialectique du même et de l'autre. Celia Rosenberg se souvient et les signes (images, scènes, noms) que lui rapporte sa mémoire sont à la fois intacts et transposés. De même, ont lieu des dédoublements entre les amants. Aux deux extrêmes de l'axe qui relie non-savoir et savoir, ils sont à la fois polarisés (opposés) et fusionnés (semblables). D'une part, il y a Karl-Heinz Hausen, figure du silence, de la négation de la vérité ( $\mathrm{d}$ 'abord silence de la langue allemande pour Celia, puis silence imposé par un pacte selon lequel aucune question concernant la vérité ne doit être posée), et d'autre part, Piotr Jalski, figure de la parole, du dévoilement de la vérité. Entre silence et parole on retrouve le musicien Schule, l'artiste comme source perpétuelle de savoir insaisissable. Par ailleurs tous se dédoublent pour rejoindre le sujet de la jouissance affirmant simultanément l'émergence des/pôles (savoir/non-savoir, vie/mort).

Ainsi, l'amour pour Karl-Heinz est hanté par les apparitions (oniriques et hallucinatoires) de la magicienne à la peau verte, obligeant constamment le présent du désir à participer de sa fin, de la mort, à en être empreint. L'ange vert est un ange de mort dont les signes demeurent indéchiffrés, inaccessibles au savoir de Celia Rosenberg. Figure du rêve prémonitoire, la magicienne à la peau verte annonce l'holocauste dans le temps même du désir inaliénable de Celia pour Karl-Heinz. Son apparition est en effet plus d'une fois superposée à celle des magnolias, forte instance érotique dans sa puissance à susciter à la fois la mémoire (réminiscence) et l'oubli (jouissance). Quant au savoir que transmet Piotr, il s'associe aussitôt à un savoir «initiatique» qui met en jeu la traversée de la mort et l'accession à la lumière (p. 150-151).

Les deux pôles du savoir et du non-savoir participent donc de l'occultation. Si le savoir est intrinsèquement lié à la mort (p. 139), le non savoir (le déni) revient à obstruer l'imminence de cette mort par du signifiant (magicienne, cartes du Tarot, Cabale). Et lorsque le savoir de la «chose» est inévitable, lorsque Piotr Jalski s'acharne à dire la vérité, Celia Rosenberg comprend alors qu'il lui faut choisir (p. 137-138), c'est-à-dire déplacer l'érotisme dans le champ du savoir: rejoindre la mort ne sera jamais que rejoindre Piotr (p. 138). Savoir sera donc encore jouir.

L'axe de l'occultation ne se maintient ainsi que de l'occultisme sur lequel se jouent deux pôles constamment dialectisés. Celui de la mémoire "trans-historique" (d'où semblent surgir les images chevalines bleues et les vies antérieures) raconte la traversée de la mort et l'impossible de la quête identitaire et de l'objet du désir. Ce pôle du signifiant dont le signifié est constamment différé apparaît donc comme celui-là même de la fiction. Pôle de l'imaginaire et de la re-présentation dans lequel l'autre pôle, celui de la mémoire historique (soirées chez Sarah de Mouliak, chez Gabrielle Lévy, enfance avec Julian, etc) finit par se fondre pour marquer la réalité au sceau de l'art: Kandinski/Schule. 
Fiction $\longleftrightarrow$ réalité recoupe donc en son point de manquement l'axe non-savoir $\longleftrightarrow$ savoir (mensonge $\longleftrightarrow$ vérité) pour en multiplier les relations. L'axe de l'occultisme oblige à inscrire les traces du réel dans le champ de l'érotisme. Érotisme de Celia Rosenberg qui s'énonce dans une symbolique de l'ange (figure ailée) refondue dans le galop coïtal et la marée bleue orgastique, préfigurée au début du roman et se développant en un réseau de plus en plus envahissant. Le bleu de la mer et le bleu du ciel (associés à l'enfance et à l'amour du frère Julian p. 30) de même que la figure chevaline (aquatique/stellaire) fondent la symbolique cosmique qui trouvera son point culminant dans la "résurrection» sur la constellation du Cygne.

Elle le chevauche avec ardeur, emportée par le feu qui la consume et embrase... Celia Rosenberg galope d'orgasme en orgasme...

Celia Rosenberg lâche prise, s'abandonne complètement à la marée montante d'or qui la galvanise. Elle se laisse aller dans la fissure qui s'ouvre en elle, crevasse bleue dans laquelle elle tourne, nautile...

On note en passant que l'allusion au feu évoque aussi la mort que connaîtra Celia.

Si l'œil vert de Karl-Heinz (ange exterminateur/bourreau) suscitait l'apparition prémonitoire de la magicienne à la peau verte, l'œil bleu des amants juifs (Klauss Schule et Piotr Jalski, anges victimes) éveille les symboles de l'érotisme qui viendront s'associer aux peaux bleues des morts/ressuscités (p. 92, 93, 138, 140,171,178). On peut par ailleurs souligner que les deux axes tournent autour d'un terme central: Auschwitz, nuit du trauma. C'est le troisième amant (Klauss Schule) qui joue un rôle particulier dans la structuration de l'écrit. Ce personnage de juif allemand traverse de façon singulière le tracé que réalisent Karl-Heinz et Piotr du mensonge à la vérité. Schule fait figure de révélateur permettant la mise en orbite des deux axes comme écriture du silence; limite du verbe encerclant le silence et s'y perdant. Il introduit l'esthétique de Kandinsky comme la clé de cet échange entre vérité et spirituel:

Klauss Schule (...) parle du mouvement Der Blaue Reiter et de l'irruption du spirituel dans l'art de Kandinsky. (...) Celia Rosenberg ne s'était pas d'abord rendue compte que Klauss Schule s'adressait à elle en allemand et qu'elle comprenait tout ce qu'il lui disait. (...) Un chéval bleu galope dans l'écume outremer de sa mémoire, traverse le temps (...) Klauss Schule se penche pour l'embrasser avec douceur sur les lèvres. Le bleu magenta de son regard lui coupe le souffle et son sexe se mouille de désir...

L'inspiration créatrice qu'expriment Klauss et Le Cavalier bleu concentre tous les aspects de la fusion entre le jouir et la mort que le savoir sous-tend. 
Savoir qui devient soudain celui de la langue allemande lorsqu'elle n'est plus que le véhicule d'une vérité artistique, c'est-à-dire déjà inscrite dans la dimension symbolique. Seuls l'art et l'érotisme sont accessibles à Celia en tant que vérité. À ce titre, il est important de reconnaître dans cette esthétique (Der Blaue Reiter) une tendance similaire à celle de Celia Rosenberg.

Ainsi, ce mouvement artistique se fondait sur une problématique de la création dans laquelle l'artiste devait rompre avec les limites de la représentation réaliste et œuvrer selon sa «nécessité intérieure». Toute cette attitude prenait ses sources dans une conception mystique du monde ${ }^{13}$. De plus, la symbolique des couleurs déployée par cette esthétique recoupe en quelque sorte l'organisation structurelle du roman de Villemaire. Un bref résumé de cette symbolique apparaît dans le Dictionnaire des symboles ${ }^{14}$ sous l'article «bleu»:

La profondeur du vert, selon Kandinsky, donne une impression de repos terrestre et de contentement de soi, tandis que la profondeur du bleu a une gravité solennelle, supra-terrestre. Cette gravité appelle l'idée de la mort: les murs des nécropoles égyptiennes étaient généralement recouverts d'un enduit bleu clair. On dit aussi des Égyptiens qu'ils considéraient le bleu comme la couleur de la vérité.

L'hippopotame de faïence bleu, objet venu tout droit de l'ancienne Égypte et autre figure chevaline aquatique («des fleuves») vient donc nouer le pacte de l'érotisme et du silence entre Celia et Karl-Heinz. Il redouble le lien vérité-mort inscrit dans le savoir de l'égyptologue et dont le discours demeure impénétrable pour Celia Rosenberg:

Il semble discourir sur l'objet. (...) Karl-Heinz continue à disserter en allemand. Elle dit: "Je ne parle pas allemand..." (...) Il reprend son discours (...) Une angoisse folle et subite vient de s'emparer de Celia Rosenberg tandis qu'elle écoute le soldat allemand discourir sur le petit hippopotame bleu dans la salle des antiquités égyptiennes.

La question obsédante qui traverse le roman (était-il égyptologue avant la guerre?) pose bien ce savoir exclusif à Karl-Heinz quant à la vérité et à la mort. L'hippopotame bleu devient donc, entre autres, le signifiant d'une passion pour une civilisation anéantie dont l'holocauste juif pourrait bien être la répétition. L'objet ancien est donc traversé par les deux axes de l'occultisme (doublement inscrit dans le culte égyptien et dans l'imaginaire de Celia) et de l'occultation. Briser l'hippopotame de faïence (p. 163) devient pour Celia l'acte destructeur marquant son accession au savoir et à la mort, ainsi que son dépassement. De même, le Sphynx, autre signifiant égyptien, instance de la vérité énigmatique et de la mort, est rejeté par Celia dans le temps même où il annonce le feu des fours crématoires. Vérité encore associée à Karl-Heinz Hausen qui est l'auteur du livre où est gravé l'animal mythologique: 
Celia Rosenberg ferme rapidement les yeux car on dirait que le livre s'enflamme et la chaleur atroce qui se dégage du lion de feu rugissant à quelques mètres dans le désert la fait se tordre de douleur.

Pour Celia, la quête n'est jamais que la recherche d'une perte, celle que produit la jouissance annihilant toute plénitude identitaire puisqu'elle en est la condition d'impossibilité. Il y a donc aussi dans la Constellation une mise en déroute des figures angéliques par le truchement de la mémoire transhistorique qui devient ainsi le point de fuite de la recherche d'identité. En effet, Celia se "souvient" qu'en des vies antérieures, l'ange exterminateurbourreau fut victime, de même que l'ange bleu-victime fut, à l'inverse, bourreau (p. 145-147).

A l'impossibilité du rapport identitaire se joint l'impossibilité du rapport sexuel soumis à l'empire de la réalité historique: impossibilité du rapport entre Celia (juive) et Karl-Heinz (soldat allemand), impossibilité du rapport entre Celia et son frère Julian, impossibilité du rapport entre Celia (épouse d'officier allemand) avec Piotr (juif polonais). Position de l'érotisme qui rejoint celle du réel, de l'indicible.

Par ce détour, on comprend que se livrer aux mains des nazis ( I Ich bin Jüden» p.158), c'est alors retourner au point aveugle de la jouissance en traversant la mort, c'est-à-dire en tentant d'écrire cet impossible à écrire, ce «je jouis» imprononçable. La fiction fait alors violence au réel, elle expulse le monde entier en un geste terminal qui revient à s'approprier entièrement le savoir sur l'extermination en en traversant toutes les étapes (chambre à gaz, four crématoire, transport, enterrement). C'est dire que le dernier bouc émissaire est devenu la réalité même. Mourir sera donc accéder à l'énonciation, au «je» qui ne peut surgir que de la position intenable du réel, du "point mort" où l'écrit combat sa propre impossibilité.

La Constellation formule la question d'un sujet pour qui la vérité est irrecevable du fait même qu'elle s'échange constamment avec le statut de la jouissance ${ }^{15}$. Mais cet échange permet que, par la traversée de la mort, la jouissance rejoigne la vérité dans cette «constellation du Cygne" où la fiction se déréalise d'accéder au réel. Chez Villemaire, l'envolée cosmique n'est donc pas l'accès à la plénitude, mais désigne cette oscillation d'un sujet travaillé par la mort et dont la constitution n'est que l'avènement d'un corps de langage où le réel fait trou.

L'écriture «hiéroglyphe» de Villemaire devient la configuration de l'indicible, tout comme, au dernier chapitre, les corps lumineux de Celia (je) et de Piotr (tu) inscrivent le point de cessation du scintillement qui les constitue. Temps du corps qui ne jouit que de se dérober:

Elle éprouve une telle confiance physique dans ce corps musclé (...) qui s'unit si parfaitement au sien qu'elle s'abandonne sans un son, dans une stase voluptueuse et duveteuse comme si un ange 
bleu aux ailes moelleuses l'emportait en la serrant dans ses bras (...) Celia Rosenberg vole au caeur de la Voie lactée blottie contre cet homme (...) leurs corps lumineux rayonnent d'énergie et d'amour.

...je meurs d'amour (...) tandis que mon corps physique n'est plus... et puis, tu es là, tout à coup, dans mon cou. Tu as mis tes mains sur mes épaules, ta joue contre la mienne.

La mort de Celia reprend ainsi la fonction du nom. De même, elle reflète l'écriture de Villemaire qui fait que l'on se tient dans l'indéfini, dans l'impossibilité de fixer les repères classiques d'une identité. Le personnage (accédant au «je») s'ouvre tout grand, s'étale, se répand:

Je meurs d'amour car je suis encore vivante, mon amour, partout sur la terre, dans d'autres corps, dans les corps de ceux et celles qui sont vivants, et dans d'autres galaxies.

L'érotisme n'est donc pas dans l'écrit. Il est dans le tracé de cette écriture qui va au corps de la lecture, dans la différence qui les sépare, dans le rythme qui scande ce passage. Ce rythme marque sa pulsation dans les noms interminablement répétés. On rencontre ici encore, comme dans la Vie en prose, ce travail de la répétition, condensé cette fois dans l'incantation des noms propres où, par l'effet de retour, quelque chose s'oublie jusqu'au nom lui-même (dernier chapitre). Le nom ramène aussi la mémoire, mais cette insistance à se rappeler (Rappelle-toi Celia Rosenberg p. 111-114) ne s'entend jamais que sur le mode de l'effacement, celui de la vérité comme celui du sujet.

C'est dans cette «absence» que revient s'inscrire la citation du Tractacus logico-philosophicus de Wittgenstein placée en exergue du roman, énonçant que tout ce qui peut être dit peut être dit clairement; et (que) ce dont on ne peut parler on doit le taire. Ce devoir-taire est la condition même du réel, défaut du "dicible» venant insister dans la mise à l'épreuve du silence par la fiction.

De la Vie en prose à la Constellation se lit bien cette épreuve dans le rapport au nom qu'entretiennent deux écritures. La première participe de l'effervescence du langage venant faire flot quand le nom manque à nommer: déferlement de la fiction que le paradoxe de l'ange restructure en point de fuite. La seconde, elle, répète l'appel du nom jusqu'à l'ouvrir sur sa prise en charge par un sujet qui en reste toujours dépossédé. De l'une à l'autre, c'est la mort sans détour ni morale, simplement là, à proximité et impossible, inconnue, qui articule tous ces processus de disparition d'identité. L'ange est bien ce point indicible où le réel s'éprouve de ne pouvoir se prouver. 
La Constellatioin est cette cruci-fiction d'un corps de jouissance dont la résurrection stellaire constitue la mise "en lumière" d'un sujet d'énonciation. Ainsi, chez Villemaire, se rejoue la "Lutte de Jacob avec l'ange», celle d'un corps à corps avec l'innommable venant mettre à nu l'inscription d'un vide qui rompt la surface d'un langage dont l'acte consiste essentiellement à renommer.

1. André Beaudet, Littérature l'imposture, Montréal, Les Herbes rouges, 1984, p. 88.

2. Yolande Villemaire, la Vie en Prose, Montréal, Les Herbes rouges, 1980.

3. Ibid., p. 98.

4. Ibid., p. 142.

5. Ibid., p. 219.

6. Ibidem, p. 93: Comment te dire que le motif de cette lettre en est un de reconnaissance? Je n'ai ni la même voix que toi ni la même voie. (...) Que tu y répondes ou pas n'a aucune importance,; p. 246: Tu as, par expérience, renoncé aux lettres qui dévient les romans. Ce n'est à personne que tu parles, à personne d'autre que toi. Tu es une autre, Vava. Tu n'es que celle, que celui qui lit.

7. Les notions de réel et de réalité ne sont plus ici synonymes comme le révèle la psychanalyse depuis Lacan. Serge Leclaire a très bien formulé cette distinction fondamentale: ... le réel est à distinguer fermement de la réalité en ce sens qu'il désigne le défaut constitutif du fait structural, alors que la réalité est faite des constructions propres à convenir le manque réel, voire à le sceller. ("Le réel dans le texte" in Littérature, no 3, octobre 1971, p. 30). Le réel? C'est ce qui résiste, insiste, existe irréductiblement, et donne en se dérobant comme jouissance, angoisse, mort ou castration. (Démasquer le réel, Paris, Seuil, coll. Points, 1971, p. 11).

8. Ibidem, p. 12: Ton nom de Los Angeles dans mon réel désert.; p. 53: C'est un ange. Je lui demande comment il s'appelle, parce que je sais déjà le nom qu'il va prononcer. Ce n'est pas tout à fait ça, mais c'est un nom d'ange.

9. André Beaudet, op. cit., p. 131.

10. La Constellation du Cygne, Montréal, Éditions de La pleine lune, 1985.

11. Ce qui rappelle un autre texte de Villemaire paru en 1982 aux Herbes rouges et qui portait le titre: Du côté hiéroglyphe de ce qu'on appelle le réel.

12. À l'article "constellation du Cygne" le Robert 2 donne: constellation boréale dont les étoiles décrivent une grande croix dans la Voie lactée.

13. Kandinsky, Du spirituel dans l'art, Denoël Gonthier, "Médiations».

14. J. Chevalier et A. Gheerbrant. Dictionnaire des symboles. T.1. Paris, Seghers, 1973, p. 210.

15. On se souvient des mots qui ouvrent Télévision de Jacques Lacan: Je dis toujours la vérité: pas toute, parce que toute la dire, on n'y arrive pas. La dire toute, c'est impossible, matériellement : les mots y manquent. C'est même par cet impossible que la vérité tient au réel. Paris. Seuil, 1974, p. 9. 


\section{BIBLIOGRAPHIE}

BEAUDET, André, Littérature l'imposture, Montréal, Les Herbes rouges, 1984.

CHEVAliER, Jean et GHEER BRANT, Alain. Dictionnaire des symboles, (4 tomes). Paris, Seghers, 1973.

DURAS, Marguerite, le Ravissement de Lol V. Stein, Paris, Gallimard, Folio, 1976.

一. le Vice-consul, Paris, Gallimard, L'imaginaire, 1977.

LACAN, Jacques, Écrits, Paris, Seuil, 1966.

—, Télévision, Paris, Seuil, 1974.

KRYSINSKI, Wladimir, "l'Intertexte du roman et l'espace comparatif" in Canadian Review of Comparativ Literature, décembre, p. 469-477.

VILLEMAIRE, Yolande, la Vie en prose, Montréal, Les Herbes rouges, 1980.

-. Du côté hiéroglyphe de ce qu’on appelle le réel, Montréal, Les Herbes rouges, [982.

-, Ange Amazone, Montréal, Les herbes rouges, 1982.

- les Coincidences terrestres. Montréal, Éditions de La Pleine Lune, 1983.

—, la Constellation du Cygne, Montréal, Éditions de La Pleine Lune, 1985. 


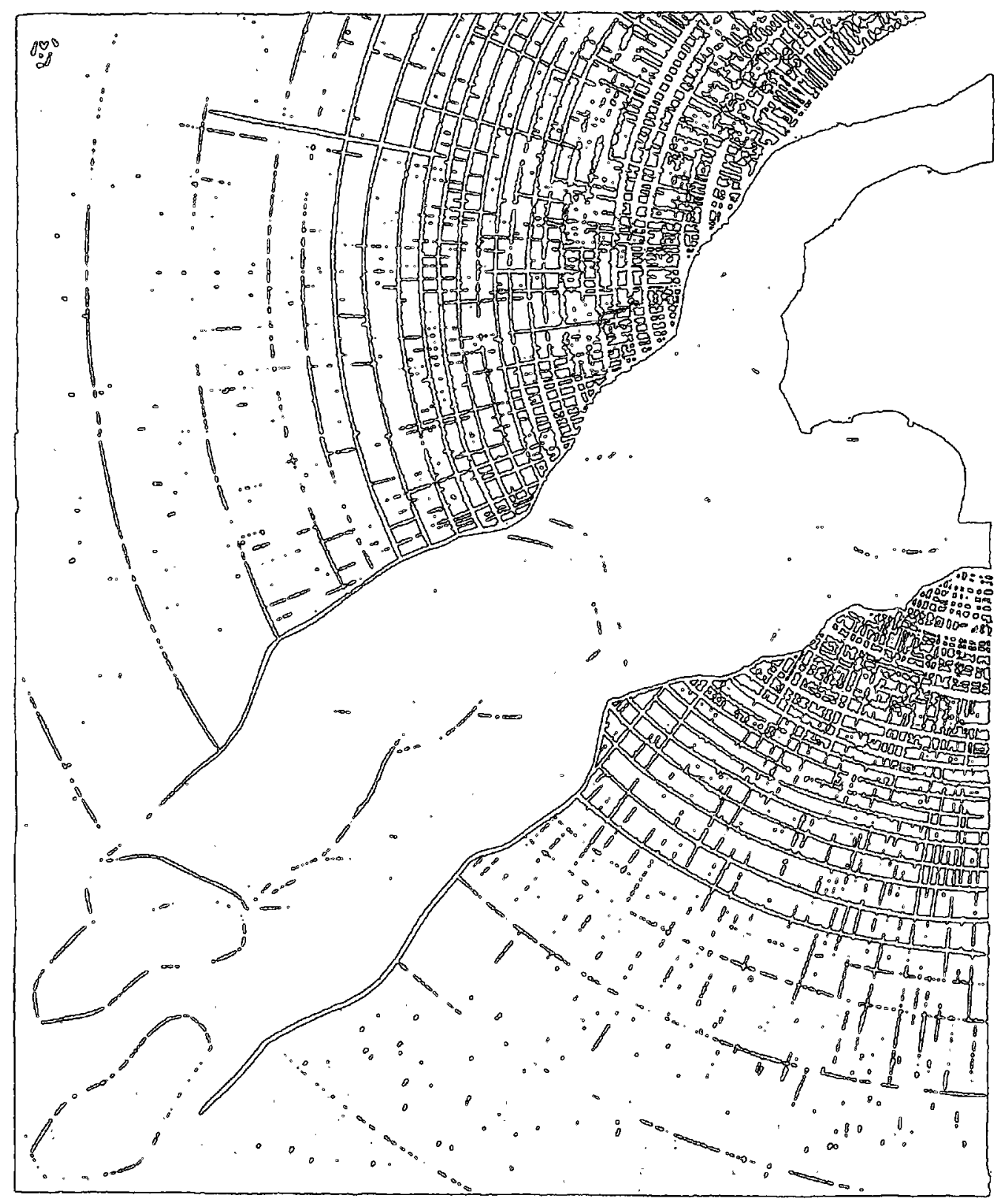

